\title{
AquaMesh - design and implementation of smart wireless mesh sensor networks for aquaculture
}

\author{
Adinya John Odey, Li Daoliang \\ College of Information and Electrical Engineering, China Agricultural University, Beijing, PRC
}

Email address:

Johnodey@yahoo.com(A. J. Odey), li_daoliang@yahoo.com(Li Daoliang)

\section{To cite this article:}

Adinya John Odey, Li Daoliang. AquaMesh - Design and Implementation of Smart Wireless Mesh Sensor Networks for Aquaculture. American Journal of Networks and Communications. Vol. 2, No. 3, 2013, pp. 81-87. doi: 10.11648/j.ajnc.20130203.15

\begin{abstract}
In this paper we design and implemented a dynamic and smart wireless mesh sensor network for aquaculture and water quality management applications. This system utilizes the Waspmote embedded systems platform developed by Libelium, mesh networking transceivers from Digi International and smart sensors from UNISM to implement a novel smart Wireless Mesh Sensor network -Aquamesh with multiple gateways of different technologies (Zigbee, GPRS and WIFI). The system is designed to continuously monitor aqua-environmental parameters and then initiate an alert or early warning to system user when certain thresholds are exceeded. The data generated from this system is stored locally on the gateway or sent to a remote web server. Data on the local database or remote web server can be accessed with smart mobile phones or personal computers. The experimental results show that the system presented in this paper is feasible to implement and present results consistent with traditional aqua-quality monitoring systems. This system will find application in the monitoring of marine and wetlands environments like fish ponds, coastal water pollution monitoring systems, effluent and sewage treatment plants, offshore oil and gas drilling facilities.
\end{abstract}

Keywords: Aqua Mesh, Wireless Mesh Sensor Networks, Aquaculture, Dynamic and Smart Gateway

\section{Introduction}

Recent advances in semiconductor technologies have presented a new paradigm within computer science: the networking of small-sized sensors which are capable of sensing, processing, and communicating. These Small and smart devices equipped with a processing unit, storage capacity, and small radios offers wireless communication and has presented new application opportunities[1]. Wireless Sensor Networks (WSN) are becoming ubiquitous and permeating every aspect of our everyday life ranging from unobtrusive applications in military and intelligence control to new areas of habitat and environment monitoring, disaster control, health care and home automation.

Wireless mesh sensor networks combine the advantages of wireless mesh networks and wireless sensor networks. As the name implies, wireless mesh sensor networks are;

- Comprised of wireless Sensor nodes. A node in this type of network consists of a sensor or an actuator that is connected to a bi-directional radio transceiver. Data and control signals are communicated wirelessly in this network and nodes can easily be battery operated.

- Arranged in a networking topology called "mesh net- work". Mesh networking is a type of network where each node in the network can communicate with more than one other node thus enabling better overall connectivity.

State of the art WMSN shares some of the following characteristics; self healing, self organizing and performs multi-hops from data source to destination. They posses decisive advantages, compared with other technologies previously used to monitor environments via the collection of physical data. The combination of these advantages enables the monitoring of phenomena of high variability, both in time and space and also makes it possible to retrieve data in real-time from locations that are difficult to access, either temporarily or permanently, thus addressing stringent responsiveness and accessibility requirements. Their capacity to organize spontaneously in a network makes them easy to deploy, expand and maintain, as well as resilient to the failure of individual measurement points.

Wireless Sensor Network (WMSN) technologies have enabled many interesting applications in pervasive and ubiquitous computing [2]. For example using wireless sensor networks in the field of Agriculture has witness a tremendous increase as practices and technologies to increase 
world food production becomes the focus of various countries as world population continues to increase. WSN in the agricultural industry find usage in wide and varied areas of applications like Precision Agriculture, vineyard monitory, irrigations and control systems, aquaculture and water quality monitoring.

Aquaculture involves cultivating freshwater and saltwater populations such as fishes, crustaceans, molluscs and aquatic plants under controlled conditions [3]. The positive impacts of aquaculture are well documented. For instance, proponents of aquaculture argue that aquaculture reduces the world dependence on wild stocks of fish, provides new jobs, and helps to feed the worlds growing population [4]. The reported output from global aquaculture operations would supply one half of the fish and shellfish that is directly consumed by humans [5]. In 2004, Aquaculture contributed more than one third of the world total production of fishes [6] and there have been consistent growth rate in production recorded over the last 30 years by different countries.[7]. For example Puerto Montt in Chile has become famous for farming and exporting Salmond and it is reported that about $90 \%$ of all the shrimps and crustaceans consumed in the United states is farmed and imported [8]. While agricultural revolution in Aquaculture is an especially important economic activity in China accounting for $70 \%$ of world production $[9,10]$, it is also becoming one of the fastest growing areas of food production in the United states.

In modern aquaculture management, water quality monitoring plays an important role. Appropriate control of the water quality to keep the concentration of the water environment parameters in the optimal range can enhance the growth rate of aquatic organisms, affect dietary utilization and reduce the occurrence of large-scale diseases incidences. Without gathering information of physical and chemical parameters of water quality together with the related ecological factors it is almost impossible to perform the appropriate water quality control at the right time in the right place.

To realize real-time data collection in a secure, robust, manageable and low-cost manner, without long-distance cable connections, is the motivation for this paper. This paper examines how best to develop a wireless mesh sensor network to provide these and other services to aquaculture farming which primarily benefits the community and enhance return on investment to the farmer. We present the research, design and development of a wireless mesh sensor network called "Aquamesh" to be deployed in an aqua cultural environment and identify some of the benefits this type of network could offer.

\section{Systems Design}

For aquaculture monitoring, we are interested in continuous monitoring of multiple environmental parameters in an aqua-culture environment through the use of wireless mesh sensor network. The parameters of interest in paper are $\mathrm{PH}$, Dissolved Oxygen (DO), Electrical Conductivity (EC) and Temperature. The systems architecture and network topology is shown in Figure 1.

\subsection{Data Generation}

Our system adopts a hybrid data generation strategy, combining all the data needs and generation requirements of time, event and query driven approach.

The system is expected to generate data values of $\mathrm{PH}$, Electrical Conductivity (EC), Dissolved Oxygen (DO) and Temperature periodically, forwarding the results to the gateway for storage in the database or to the internet server when available. This allows for the best energy consumption profile, because radio resources are used only when it is considered necessary by the application.

Also if the parameters exceeds or fall below the thresholds of 6.0 or 8.5 for PH, 20mg/1 for DO, $200 \mu \mathrm{s} / \mathrm{cm}$ for $\mathrm{EC}$ and $340 \mathrm{C}$ for temperature, short message service (SMS) alert are sent to the farmer or administrators to enable instant remedial actions to be initiated. This allows capture on the flight important events, whose freshness is important for the proper operation of the system.

The design also allows for users generated queries to be performed on the sensors at any time to acquire needed data. These queries can be generated through any of the WIFI, GPRS/SMS or ZigBee gateway.

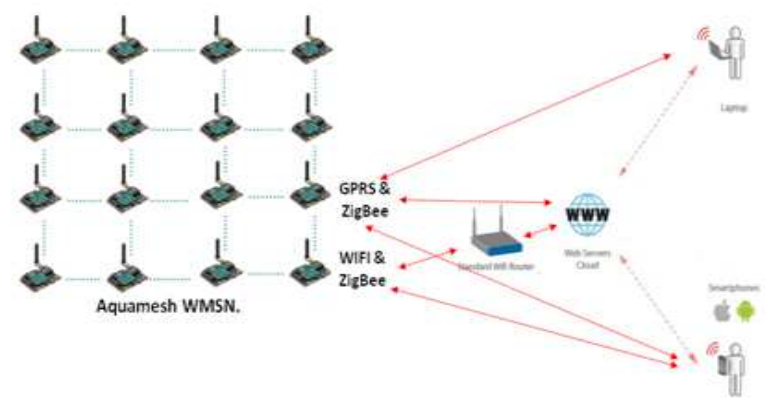

Figure 1. Systems architecture of Aquamesh.

\subsection{Data Transport}

We envisioned a wireless meshed sensor network where data generated in the field can be accessed and processed in the comfort of a remote office. We consider a direct connection through a single gateway station unfeasible due to the remote locations of most farms. On the other hand, a pure complete mesh network, where full connectivity is required, would not be the case in this design because of the complexities and resources utilization of such a topology. In this system, a partial wireless mesh network topology is utilized to achieve data transport from the end nodes through intermediate nodes to the gateways and finally the remote user's location.

To accomplish high data throughput and availability, prompt access to data from remote location, to ensure maximum reliability and avoid data loss, the system is designed with multiple gateways using a mix of technologies 
(GPRS, WIFI, ZigBee) to ensure sensed data is communicated to the end user.

\section{Systems Implementations}

Aquamesh is composed of (a) Wireless Mesh sensor nodes, (b) a GPRS/SMS gateway, (c) a WIFI gateway, and a network implementation strategy using (d) mesh topology to transport data generated from (e) smart sensor probes to (f) remote web server $(\mathrm{g})$ smart mobile phones or a personal computer fitted with (h) ZigBee dongle gateway.

\subsection{Wireless Mesh Sensor Node}

The wireless mesh sensor node implemented is a Waspmote embedded systems board integrated with Atmega 1281 micro controller unit (MCU) [11], a wireless mesh network transceiver radio module (DigiMesh 2.4 transceiver radio chip) [12], smart sensors [13, 14, 15] for PH, EC, DO and temperature connected through a Waspmote [16] Prototyping board [17] and a battery power source complemented with an energy harvesting solar module [18].

Waspmote does not support RS485 smart sensors either directly or through the Prototyping board. To implement our Aquamesh WMSN, an RS485 to RS232 UART conversion was done using CAU-RTU-0-01 chip module. Smart Digital Sensor probes CAU-PH-3000, CAU-DO-1100, and CAU-EC-4000 for PH, DO, and EC respectively developed by UNISM were used to acquire data and communicate on request from the MCU using the Unibus Protocol. These sensors are connected to the Waspmote node through the RS485 interface of the CAU-RTU-0-01 chip .The prototyping board provides $+3.2 \mathrm{~V}$ power which powers all the Sensors. As part of the design to enable low power consumption and individual control of the sensors, the software ensures the sensors are powered serially one after another. This allows only one sensor to be active at a time.

The architecture of Aquamesh is designed to have dynamic and multiple gateways implementing a mix of wireless technologies as shown in figures 1 to ensure reliability and access to data generated at any time. Figure 3 shows the Aquamesh node with Digimesh transceiver module, Sensor boards and smart sensors integrated in Waspmote board. The technologies used for the gateway are GPRS, WIFI and Zigbee as shown in Figure 3, Figures 4 and Figures 5 .

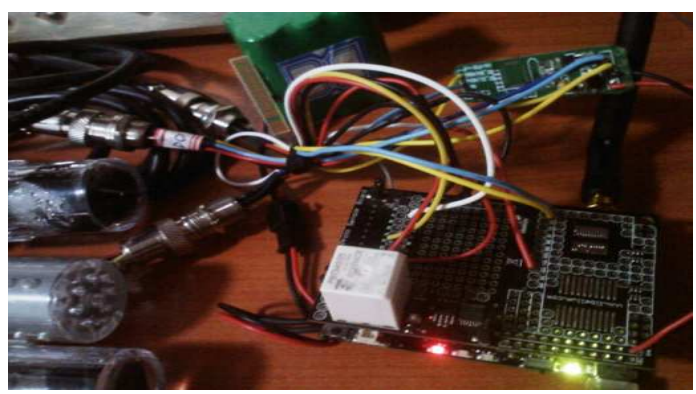

Figure 2. Aquamesh WMSN node

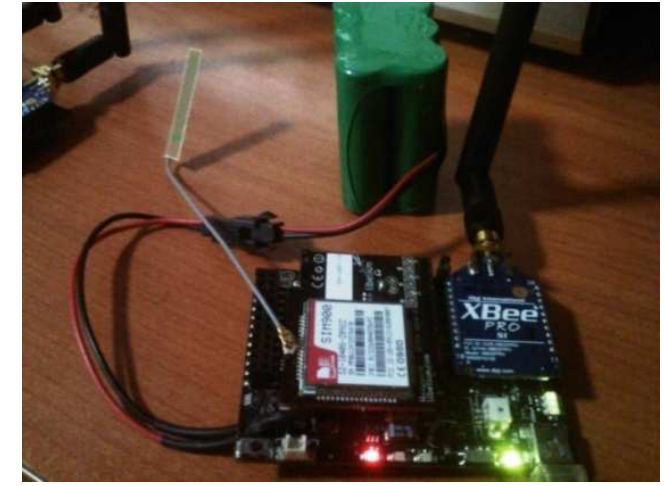

Figure 3. GPRS/SMS gateway nodes

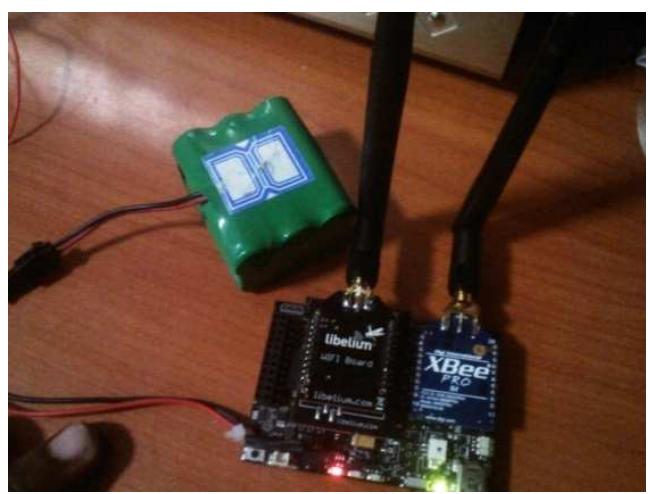

Figure 4. WIFI gateway nodes

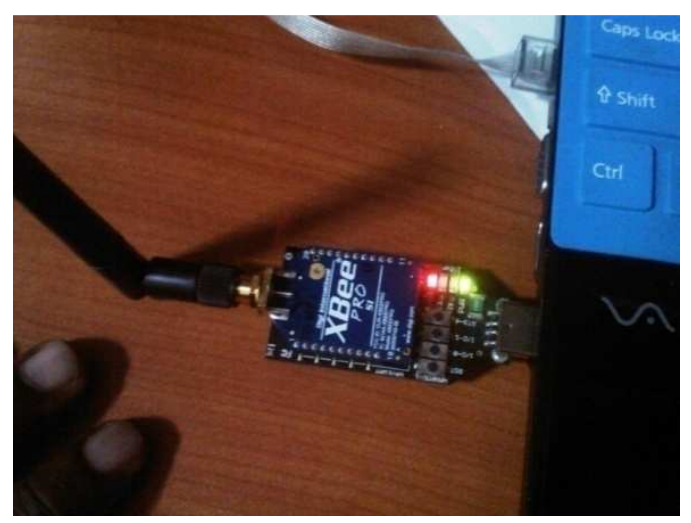

Figure 5. Zigbee gateway

\subsection{Power Source}

The design for Aquamesh WMSN requires the infrastructural mesh nodes to be powered continuously. In this system the radio devices account for most of the power consumption.

The current design utilizes a battery pack of three rechargeable nickel metal hydride (NiMH) AA type batteries which produces over 4.2 volts and has a capacity of $2.5 \mathrm{Ah}$. In order for the mesh node to be continuously powered, it requires a supplement power source derived from renewable sources of solar. This supplement power source can be used to charge the batteries and power the mesh node. Figure 6 shows the solar panel and casing for the implemented Aquamesh node. 


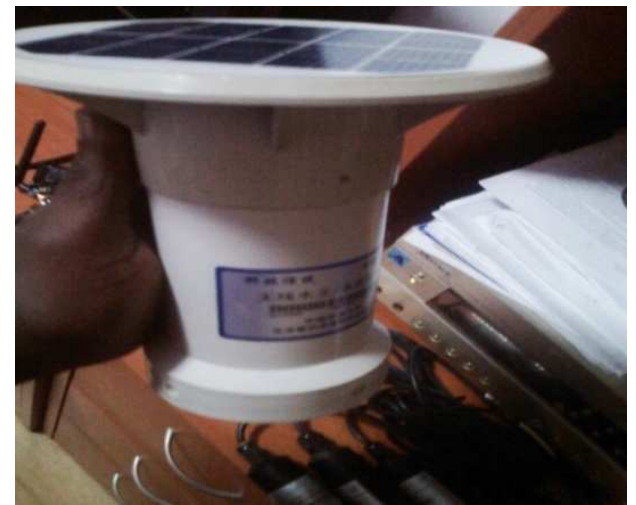

Figure 6. Solar Panel and final housing for Aquamesh

\subsection{Software}

Aquamesh software implementations cut across several platforms. From $\mathrm{C}$ and Java programming languages in the embedded Waspmote hardware to internet explorers and Android platforms for implementations of the mobile interface, the programming implementation is flexible and powerful enough to allow fast deployment.

\subsubsection{WaspMote IDE}

The Waspmote-IDE [199] (Integrated Development Environment) is used to program Waspmote. It is based on open source Arduino platform compiler, following the same style of libraries and operation. It is the interface for programming as well as controlling the Waspmote.

\subsubsection{Web Interface}

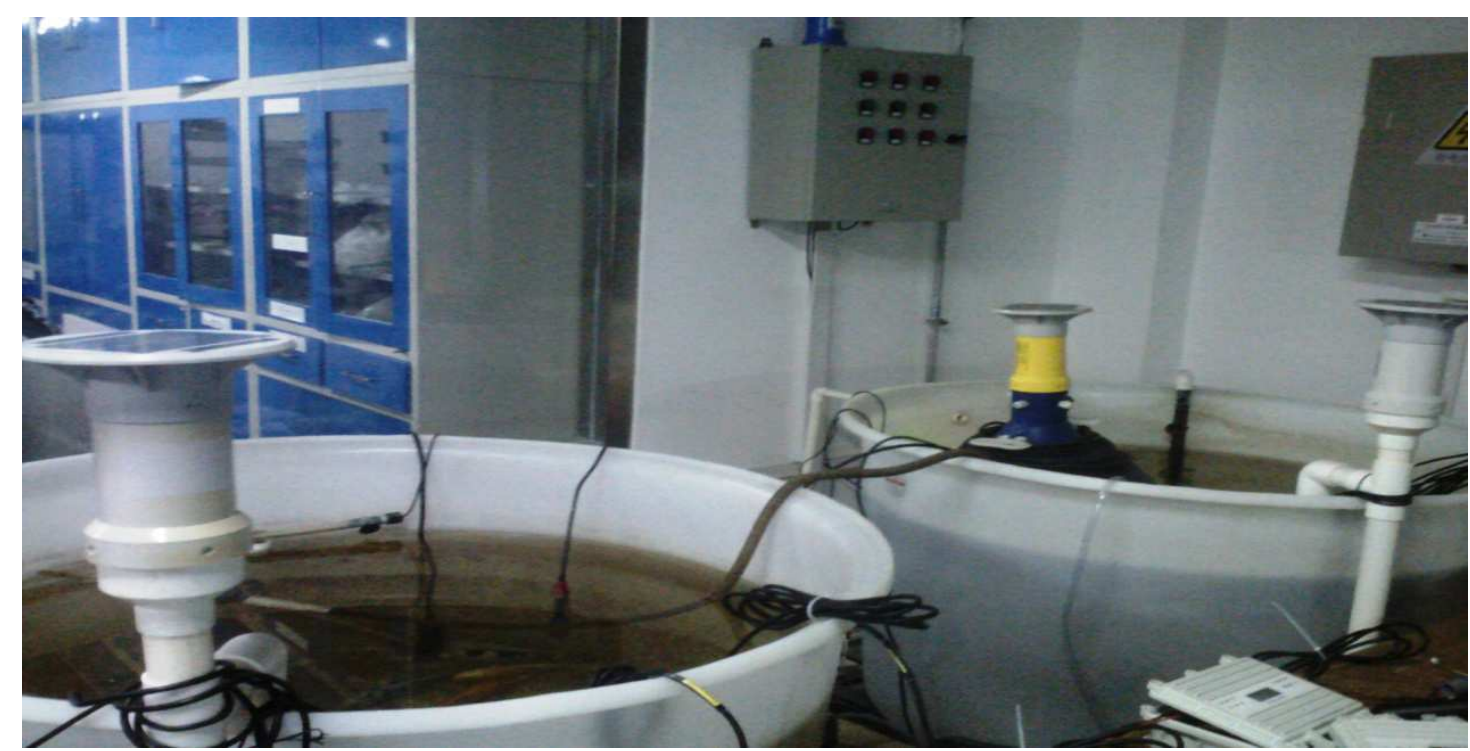

Figure 7. Experimental Deployment of Aquamesh in Fish Pond

The pond equipped with aerator machines and had thirty Koi fishes breeding. The Sensors were set to acquire data
Part of the system design is to store and display data generated from Aquamesh on a remote web server where it will be accessed with an ordinary internet explorer. To validate this functionality, the data generated from the network between 16th February, 2013 and 17th March, 2013 was sent once a day to the cloud web server hosted by Digi International [18]. The system support the use of SMS messages as well as analysis of data; the status of the network, graphs and Network diagram as well as records of the data generated can be accessed from the webpage. Data update to the web server is received through the GPRS/SMS or WIFI gateways.

\subsubsection{Android Mobile Phone}

The designs of this system also include the use of smart phones to send control commands, access and analyze data. The Smartphone is expected to pair with the WIFI gateway in an adhoc network topology or as a client through an access point (AP).

\section{Testing and Results}

To validate the designs and implementations of Aquamesh Wireless Mesh Sensor Networks, six nodes were deployed in an aquaculture fish pond at the Laboratory of Internet of Things a China Agricultural University from February 16th, 2013 to March, 14th, 2013. Three of the nodes served as data acquisition nodes each fitted with three smart sensor probes described in section 4 . The three other nodes serve as the gateway nodes with the GPRS/SMS node made the primary gate way over WIFI and ZigBee gates. 

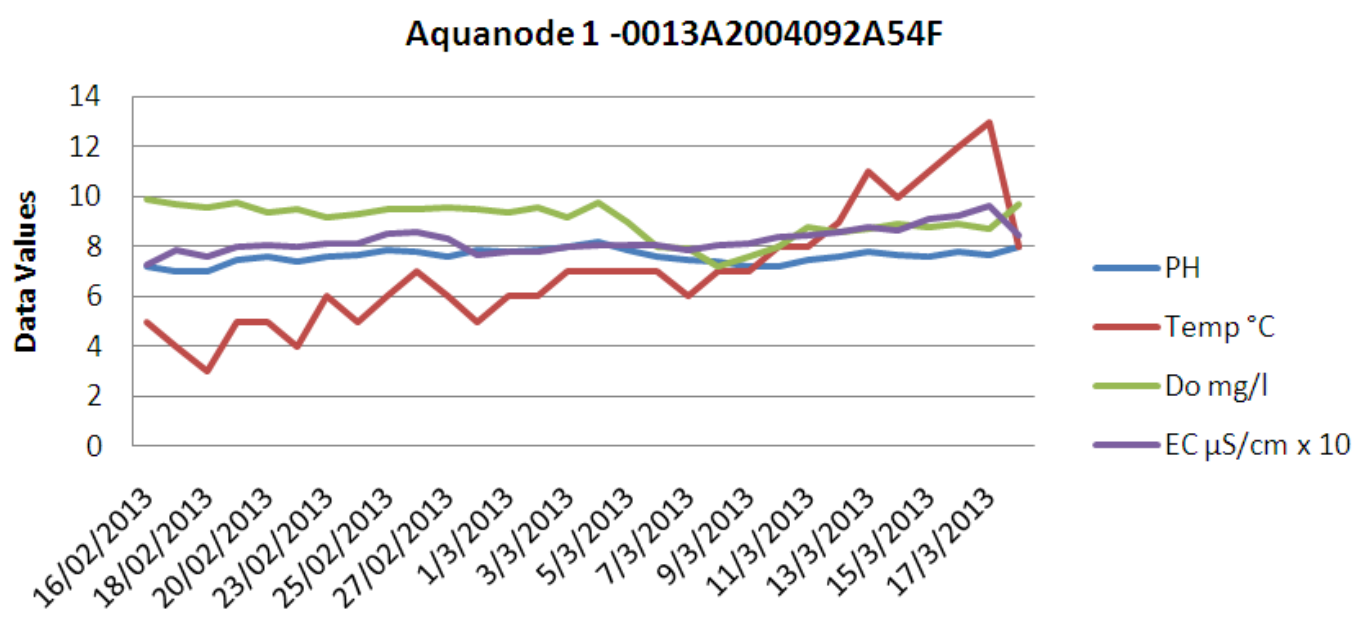

Figure 8. Test Sensors readings from Aquamesh node 1

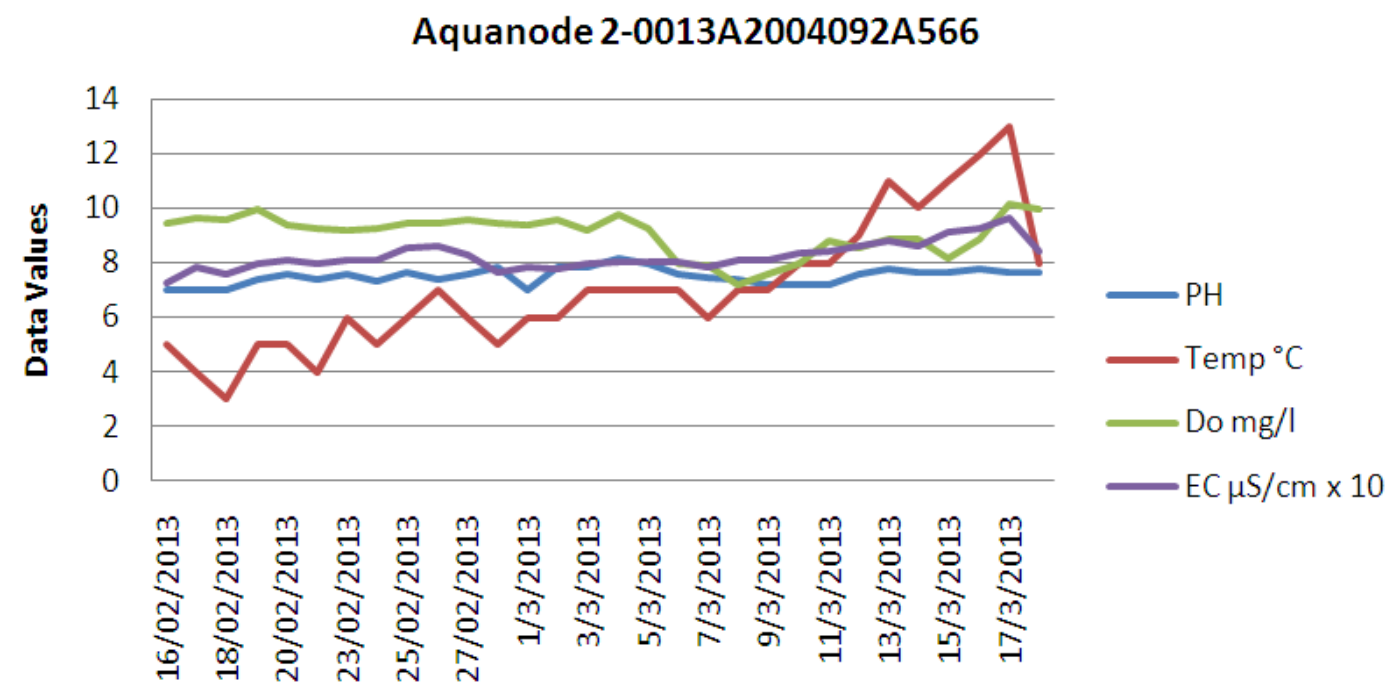

Figure 9. Test Sensors readings from Aquamesh node 2

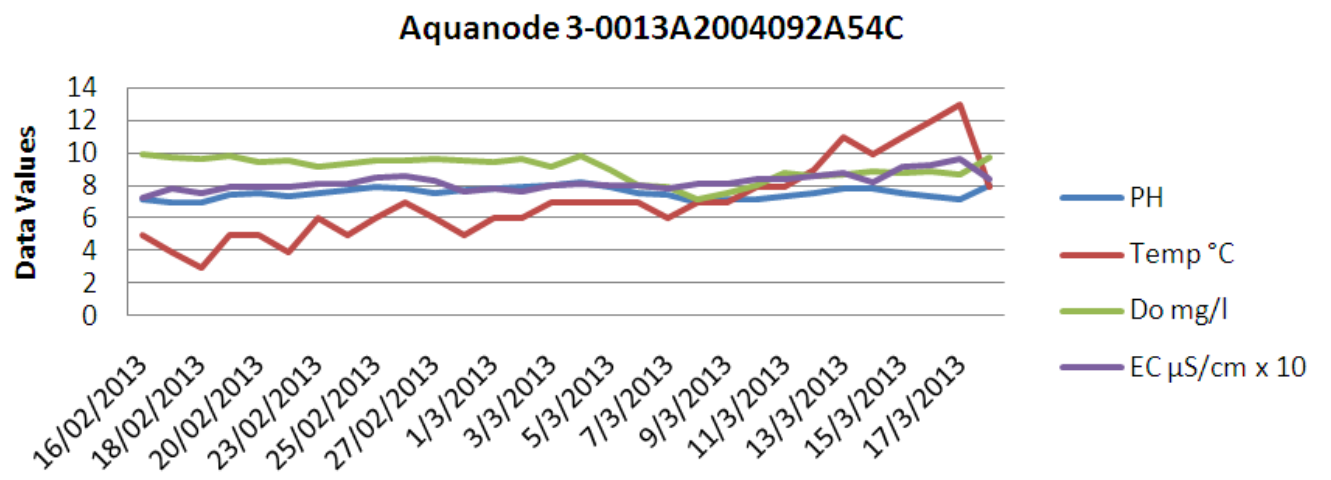

Figure 10. Test Sensors readings from Aquamesh node 3

\subsection{Data Acquisition}

Figure 5.4- Figure 5.6 shows the sensor readings of DO, $\mathrm{pH}, \mathrm{EC}$ and Temperature from the three nodes. The graph shows the $\mathrm{pH}, \mathrm{EC}, \mathrm{DO}$ concentration and water temperature trends from February 16th to March 14 . We can see the periodic change of the water temperature every day, but DO concentration only fluctuated around $9 \mathrm{mg} / \mathrm{l}$ before March 5th. The DO concentration of March 7th, March 8th and March 9 is lower than the other days because the aerator machine was manually turned off. After March 10th, the Aerator machine returned to work normal and DO concentration return to previous range. Also an SMS alert for DO 
was sent out from the three nodes on the 8th of March since the threshold for alarm was set for $7.3 \mathrm{mg} / \mathrm{l}$. It is also observed that the EC values tend to rise with increase in temperature. The reason for this trend is outside the scope of this work. The data for $\mathrm{pH}$ sensors are also shown. The values from the three nodes fall within the range of 6.7 to 8.7 and this shows a trend consistent with $\mathrm{pH}$ range in aquaculture environments. The gateways functionalities were validated by changing the Hierarchy of the three nodes alternatively. The data transported through the ZigBee gateway was captured using the WaspMote IDE programming interface while data sent through the WIFI and GPRS/SMS were captured using the remote web server of Digi Cloud. Results captured through the three gateway nodes shows consistencies in accuracy; however the ZigBee gateway seems to handle data transfer faster than the other technologies. These could be as a result of routing paths used by the different technologies to send data to the server.

\subsection{Node and Route Discovery Test}

This test is to verify the basic operation to initialize the mesh nodes with a hop count and routing information. The nodes were placed in discovery mode. A discovery command was sent from the PC through the Zigbee Gateway to the network using X-CTU from Digi International. This command was relayed as a broadcast by the gateway node. Node 2 was positioned to be the only node in RF range of the gateway. Node 2 then relayed this discovery broadcast to Node 1 which in turn relayed it to Nodes 3 .

This test is configured so that all nodes would report their hop count and routing data to the gateway node after 2 seconds. This test was verified using X-CTU to monitor the data packets received by the PC. All nodes were correctly initialized.

Also if the source node doesn't have a route to the requested destination, the packet is queued to await a route discovery (RD) process. This process is also used when a route fails. A route fails when the source node uses up its network retries without ever receiving an ACK. Figure 10 shows the network route map.

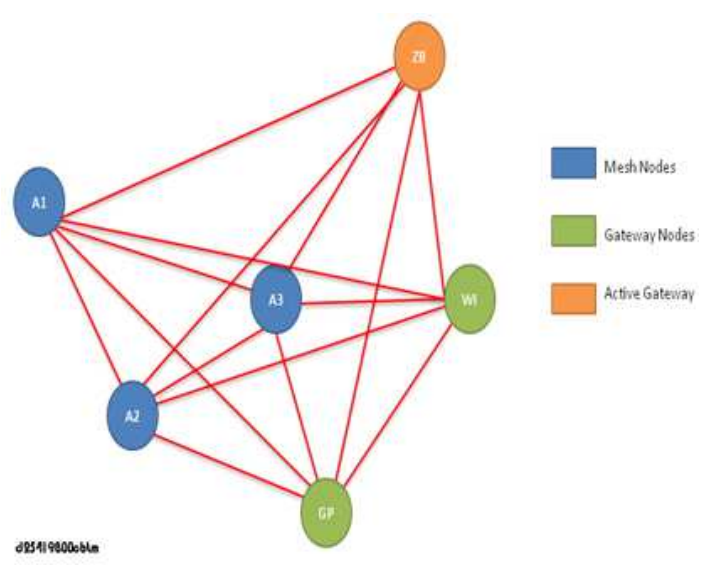

Figure 11. Network Route Map

\section{Conclusion}

In this paper, we presented the design and implementations of a smart, wireless mesh sensor network suitable for use in data acquisition in Aqua culture. The system implemented was deployed to continuously monitor water chemistry in a fish pond. Aquamesh is able to initiate an alert when certain thresholds are exceeded and the data generated was successfully sent to a remote web server for storage and processing or directly to human in the loop for remedial actions. The data generated show consistency with data generated using other methods of aqua culture measurements.

One of the key design features in this paper is the use of multiple, dynamic gateway nodes. These gateway nodes were implemented using a mixture of wireless technologies like GPRS/SMS, WIFI, and Zigbee. The system is designed to consume little power, generate data and transport data more efficiently to remote locations. The system presents the advantages of mesh networking and the simplicity of using a modular approach to systems designs.

\section{References}

[1] Marcel Busse."Algorithms for Energy Efficiency in Wireless Sensor Networks". PhD Dissertation, 2007. Accessed on 26/07/2012. Available at http://d-nb.info/987608029/34J. Clerk Maxwell, A Treatise on Electricity and Magnetism, 3rd ed., vol. 2. Oxford: Clarendon, 1892, pp.68-73.

[2] Holger Karl, and Andreas Willig," Protocols and Architectures for Wireless Sensor Networks. John Wiley \& Sons, 2005, pp.15-329. doi:10.1002/0470095121

[3] Aquaculture's growth continuing: improved management techniques can reduce environmental effects of the practice.(UPDATE)." Resource: Engineering \& Technology for a Sustainable World 16.5 (2009): 20-22. Gale Expanded Academic ASAP. Web. 1 October 2009. http://find.galegroup. com/gtx/start.do?prodId=EAIM.

[4] Half Of Fish Consumed Globally Is Now Raised On Farms, Study Finds Science Daily, September 8, 2009.

[5] FAO (2006) The State of World Fisheries and Aquaculture (SOPHIA)

[6] Blumenthal, Les (August 2, 2010). "Company says FDA is nearing decision on genetically engineered Atlantic salmon". Washington Post. Retrieved August 2010.

[7] The State of World Fisheries and Aquaculture (SOFIA) 2004

[8] Wired 12.05: “The Bluewater Revolution." http://www. wired.com/wired/archive /12.05/fish_pr.html. Accessed August 12th, 2010.

[9] Eilperin, Juliet (2005-01-24). "Fish Farming's Bounty Isn't Without Barbs". The Washington Post.

[10] Waspmote Technical Guide http://www.libelium.com/upl oads/2013/02/waspmote-technical_guide_eng.pdf. Accessed on $2013 / 03 / 15$.

[11] Digi International "XBee/XBee-PRO®DigiMesh $2.4 \mathrm{RF}$ 
Modules data sheet".http://www.digi.com/products/wir eless-wired-embedded-solutions/zigbee-rf-modules/zigbeemesh-module/xbee-digimesh-2-4\#docs. Accessed on 2013/ $03 / 15$.

[12] Yaoguang Wei, Qisheng Ding, Daoliang Li, Haijiang Tai, and Jianqin Wang "Design of an Intelligent Electrical Conductivity Sensor for Aquaculture Sensor Lett. 2011, Vol. 9, No. 3 1546-198X/2011/9/1044/005 doi:10.1166/sl.2011.1395

[13] Qisheng Ding, Haijiang Tai, Daokun Ma, Daoliang Li and Linlin Zhao. "Develop ment of a Smart Dissolved Oxygen Sensor Based on IEEE1451.2" Sensor Lett. 2011, Vol. 9, No. 3 1546-198X/2011/9/1049/006 doi:10.1166/s1.2011.1397

[14] Haijiang Tai, Qisheng Ding , Lihua Zeng, Shuangyin Liu , Daoliang Li An intelligent ammonia sensor based on mul- ti-parameter for aquaculture” Sensor Lett. 2011, Vol. 9, No. 3 1546-198X/2011/9/1049/006 doi:10.1166/s1.2011.1397

[15] Waspmote Prototyping Board http://www.libelium. com/uploads/2013/02/prototyping-sensor-board 2.0. Accessed on $2013 / 03 / 15$.

[16] Akyildiz, I.F., Melodia, T., and. Chowdhury, K., A survey on wireless multimedia sensor networks. Computer Networks,vol.51, issue4: p. 921-960, 2007.

[17] "X-CTU Configuration \& Test Utility Software". http://ftp1. digi.com/support/documentation/90001003_A.pdf. Accessed on February $2^{\text {nd }}, 2013$.

[18] Etherios, "Device cloud". https://my.idigi. com/home.do\#. Accessed on May $2^{\text {nd }}, 2013$. 